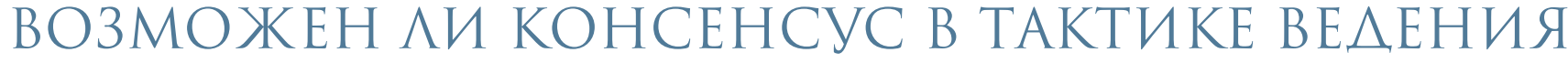 РАННЕГО ВОССТАНОВИТЕАЬНОГО ПЕРИОАА ПОСАЕ ПОЯСНИЧНОЙ МИКРОАИСКЭКТОМИИ?
}

\author{
Н.И. Турсынов, М.А. Григолашвили, Н.И. Шевелева, Ш.С. Муратбекова \\ Медииинскии́ университет Караганды, Караганда, Казахстан
}

\begin{abstract}
Цель исследования. Анализ различий в междисциплинарных подходах по отношению к срокам возвращения пациентов к повседневной двигательной активности после неосложненной поясничной микродискэктомии и готовности врачей разных специальностей к работе по единым рекомендациям.

Материал и методы. Проведено письменное анкетирование 60 специалистов (20 нейрохирургов, 28 неврологов и 12 реабилитологов), участвующих в ведении пациентов в течение первых 6 мес. после неосложненной поясничной микродискэктомии. Использована анкета, включающая 12 вопросов с несколькими вариантами ответов о сроках возвращения к повседневной двигательной активности, необходимости предоставления больным письменных рекомендаций по ограничениям в двигательном режиме, физической работе, сексуальной активности, а также ортезирования.

Результаты. Продемонстрирован значительный диссонанс в тактике послеоперационного ведения пациентов по рекомендуемым срокам возвращения к повседневной активности между врачами как одной специальности, так и разных специальностей. Все опрошенные сочли полезным создание единых письменных рекомендаций по двигательному режиму, 90 \% специалистов готовы использовать предложенные рекомендации после небольшой доработки. Отмечено некоторое увеличение рекомендуемых сроков возвращения к повседневной двигательной активности в сравнении с рекомендациями врачей других стран.

Заключение. Продемонстрированные междисциплинарные различия в лечении, реабилитации и сроках ограничения двигательной активности пациентов после поясничной микродискэктомии требуют унификации тактики послеоперационного ведения.

Ключевые слова: боль в спине, грыжа диска, нейрохирургия, микродискэктомия, реабилитация.
\end{abstract}

Аля цитирования: Турсынов Н.И., Григолашвили М.А., Шевелева Н.И., Муратбекова Ш.С. Возможен ли консенсус в тактике ведения раннего восстановительного периода после поясничнои́ микродискэктомии? // Хирургия позвоночника. 2020. Т. 17. № 2. С. 43-48.

DOI: http://dx.doi.org/10.14531/ss2020.2.43-48.

\section{IS IT POSSIBLE TO OBTAIN CONSENSUS ON THE TACTICS OF EARLY REHABILITATION PERIOD AFTER LUMBAR} MICRODISCECTOMY?

N.I. Tursynov, M.A. Grigolashvili, N.I. Sheveleva, Sh.S. Muratbekova

Karaganda Medical University, Karaganda, Kazakhstan

Objective. To analyze distinctions in multidisciplinary approaches with respect to the timing of patients returning to daily physical activity after uncomplicated lumbar microdisectomy and to the readiness of doctors of various specialties to work according consensus guidelines. Material and Methods. A written questionnaire survey of 60 specialists ( 20 neurosurgeons, 28 neurologists and 12 rehabilitologists) involved in the management of patients during the first six months after uncomplicated lumbar microdisectomy was conducted. The questionnaire included 12 questions with several answer options on the timing of returning to daily physical activity, and on the need to provide patients with written recommendations on limitations in motion regimen, physical work, sexual activity, and bracing.

Results. Significant dissonance both between doctors of the same specialty and of different specialties was demonstrated in the tactics of postoperative management of patients regarding the recommended terms for returning to daily activity. All respondents found it useful to create unified written recommendations on the motion regime, $90 \%$ of specialists are ready to use the proposed recommendations after some modification. There was a slight increase in the recommended timing for return to daily physical activity compared with the recom mendations of doctors in other countries.

Conclusion The demonstrated interdisciplinary differences in the treatment, rehabilitation and timing of physical activity restriction for patients after lumbar microdisectomy require unification of the tactics of postoperative management.

Key Words: back pain, disc herniation, neurosurgery, microdisectomy, rehabilitation.

Please cite this paper as: Tursynov NI, Grigolashvili MA, Sheveleva NI, Muratbekova ShS. Is it possible to obtain consensus on the tactics of early rehabilitation period after lumbar microdiscectomy? Hir. Pozvonoc. 2020;17(2):43-48. In Russian.

DOI: http://dx.doi.org/10.14531/ss2020.2.43-48. 
В современном обществе неуклонно увеличивается количество пациентов, подвергшихся микродискэктомии на пояснично-крестцовом отделе позвоночника, а исходы таких операций в значительной степени зависят от послеоперационной реабилитационно-восстановительной тактики. Высокая распространенность патологии, длительность временной потери трудоспособности и возможная инвалидизация пациентов придают проблеме не только медицинский, но и социальный аспект.

Несмотря на большое количество исследований по эффективности реабилитационных мероприятий после микродискэктомии, в клинической практике наблюдаются значительные различия в содержании, продолжительности и интенсивности восстановительных программ, а сроки возвращения к привычной активности варьируют в зависимости от страны лечения, больницы и даже лечащего врача [1, 2]. В литературе обсуждаются различия в сроках реабилитации, рекомендуемых нейрохирургами [3-6], однако их сопоставление с рекомендациями других специалистов мультидисциплинарной бригады (неврологов, реабилитологов) отсутствует.

Цель исследования - анализ возможности создания единых междисциплинарных и внутридисциплинарных рекомендаций по срокам возвращения к повседневной двигательной активности после неосложненной поясничной микродискэктомии на примере Республики Казахстан.

\section{Материал и методы}

Проведено анкетирование 60 врачей (20 нейрохирургов, 28 неврологов, 12 реабилитологов) города с населением около 500 тыс. человек. Время проведения анкетирования - сентябрь 2019 г.; среднее время, затраченное каждым респондентом на заполнение анкеты, - 8-10 мин. Все опрошенные являются практикующими врачами, работающими в государственных медицинских учреждениях (стационаpax и/или поликлиниках), участвующи- ми в ранней реабилитации пациентов после поясничной микродискэктомии. Предшествующий опыт работы врачей не учитывался.

Для опроса разработана анкета, состоящая из 12 вопросов, где предлагалось выбрать один из нескольких вариантов ответов, касающихся сроков возвращения к повседневной двигательной активности и необходимости предоставления больным после поясничной микродискэктомии письменных рекомендаций по ограничению пребывания в положении сидя, подъема тяжестей, вождения, скручиваний в позвоночнике, сидячей и физической работы, сексуальной активности, а также необходимости фиксации позвоночника. Все вопросы являлись прямыми, открытыми или закрытыми, направленными на получение непосредственной информации.

В ходе исследования проведен анализ анкет и интерпретация полученных данных.

Также респондентам был предложен вариант рекомендаций, разработанных на кафедре неврологии, нейрохирургии, психиатрии и реабилитологии Медицинского университета Караганды (Казахстан) на основе данных литературы 2018-2019 гг. [7].

1. Положение сидя. В течение первого месяца после операции рекомендуется не сидеть. При мочеиспускании и/или дефекации необходимо присаживаться, опираясь на унитаз полусогнутыми руками. Со второго месяца разрешено сидеть, начиная с 5 мин в день, постепенно увеличивая продолжительность до 60 мин 2-3 раза в день к 6-й неделе после операции. Пребывание в положении сидя более 8 ч в сутки рекомендовано не ранее чем через 2 мес. после операции. При длительной работе в положении сидя необходимо сохранять естественные шейный и поясничный лордозы (изгибы). Для этого стул должен повторять контуры спины, с валиками на уровне шеи и поясницы. Высота стула и стола подбирается индивидуально, с учетом сохранения физиологического положения позвоночника, с исключением чрезмерного сгибания и скручивания.
2. Положение стоя. В течение первого месяца после операции рекомендуется длительно не стоять на одном месте.

3. Рекомендуется использовать безопасный двигательный стереотип. При любых движениях необходимо удерживать лордоз (изгиб) в пояснице, сохранять плечи и таз в одной плоскости, избегая сгибания, разгибания и скручивания в пояснице.

4. Глубокие наклоны и скручивания в позвоночнике рекомендуются не ранее чем через 1 мес. после операции. При необходимости скручивания использовать поворот всем туловищем. При необходимости глубокого наклона присаживаться и вставать с опорой на ноги. Вставая из положения лежа, использовать переход через положение на боку, избегая скручивания.

5. Подъем тяжестей. В течение первого месяца после операции рекомендуется не поднимать вес более 3 кг одной рукой и более 6 кг одновременно двумя руками. При поднятии тяжестей рекомендуется не нагибаться, а с прямой спиной присесть на корточки и вставать, используя силу ног.

6. Повседневная активность. В течение первого месяца после операции рекомендовано избегать тяжелой работы по дому (длительной уборки пылесосом и мытья полов, ручной стирки и длительного мытья посуды в положении стоя и т.д.). В дальнейшем нужно постепенно расширять повседневную активность в зависимости от самочувствия, при необходимости дать спине отдохнуть в положении лежа на спине или на боку с согнутыми ногами.

7. Сексуальная активность. В течение 1-го мес. после операции рекомендуется избегать интенсивной сексуальной активности, в дальнейшем - положений, вызывающих боль в спине, при необходимости использовать подушки под поясницу.

8. Вождение автомобиля. Рекомендуется не управлять автомобилем в течение 1-го мес. после операции.

9. Трудоспособность. Возврат к работе зависит от вида двигательной нагрузки, рекомендован не ранее чем через 4-6 недель после опера- 
ции. При работе, связанной с длительным пребыванием в положении сидя (более 8 ч в день), - не ранее чем через 2 мес. после операции.

10. Спорт. Через 1 мес. после операции рекомендуется начать аэробную симметричную тренировку не менее 30 мин в день 3 раза в неделю (плавание, скандинавская ходьба, лыжи и т.д.). Избегать тяжелой и резкой физической нагрузки (прыжков, подъ ема штанги или гантелей с большим весом и др.), а также несимметричных видов деятельности (тенниса и др.). Рекомендованы упражнения для мышц брюшного пресса и длинные разгибатели спины без чрезмерного сгибания и/или скручивания в позвоночнике.

11. Ортопедический корсет. В течение 2 мес. после операции рекомендуется ношение полужесткого ортопедического корсета при физической нагрузке.

\section{Результаты}

Результаты опроса с распределением ответов специалистов представлены в табл., которая иллюстрирует существенные различия в структуре ответов между специалистами практически по всем заданным вопросам. При этом во всех практических рекомендациях, касающихся активности пациентов, нейрохирурги склонны их ограничивать в наименьшей степени.

Так, по вопросам 1-4 подавляющее большинство нейрохирургов (от 90 до 100 \%) ограничивают нормальное функционирование пациента не более чем на 2 мес. Неврологи и реабилитологи в своих рекомендациях существенно более осторожны, особенно в отношении поднятия тяжестей, вождения автомобиля и скручивающих нагрузок.

От 80 до 95 \% нейрохирургов считают возможным возврат к работе (сидячей и физической) к 4-му мес. после операции. В свою очередь, более половины реабилитологов и неврологов считают, что возврат к сидячей работе возможно только через 5 мес. после операции, а к физической - через 6 мес.
Условный консенсус между специалистами отмечен в отношении преимуществ полужесткого ортопедического корсета перед жестким и длительности его использования, причем реабилитологи в этом вопросе оказались даже более активны, чем другие специалисты, считая в подавляющем большинстве достаточным сроком для его применения 2 мес. (неврологи и нейрохирурги рекомендуют использовать ортез 3 мес.).

До 90 \% нейрохирургов рекомендовали возвращаться к сексуальной активности в течение 1 мес. после операции. Подавляющее большинство (75\%) неврологов придерживается той же позиции, а большинство реабилитологов считает возможным возвращение к данному виду активности не раньше чем через 2 мес.

Большинство специалистов предоставляет пациентам письменные рекомендации по двигательному режиму, при этом только среди нейрохирургов есть те, кто предпочитает этого не делать.

Все опрошенные сочли полезным создание единых письменных рекомендаций по двигательному режиму, при этом предложенный респондентам вариант готовы использовать в практической деятельности большинство реабилитологов и неврологов, в отличие от нейрохирургов, среди которых 60 \% считают необходимой их доработку.

\section{Обсуждение}

На момент данного исследования проведение реабилитации в Казахстане регламентировалось несколькими нормативными документами (перечислены по датам введения в действие): «Об утверждении стандарта организации оказания медицинской реабилитации населению Республики Казахстан» (Приказ Министра здравоохранения РК от 27.12.2013 г. № 759); «Об утверждении Правил восстановительного лечения и медицинской реабилитации, в том числе детской медицинской реабилитации» (Приказ Минздравсоцразвития РК от 27.02.2015 г. № 98), а также Клиническими протоколами 1, 2 и 3-го этапов медицинской реабилитации по профилю «Неврология и нейрохирургия» (взрослые)» от 15.08.2016 г. Непосредственно перед исследованием был введен «Стандарт организации оказания медицинской реабилитации населению Республики Казахстан» (Приказ М3 РК № Р ДСМ-120 От 29.08.2019 г.), a c 13.02.2020 г. действует Приказ М3 РК КР ДСМ-9/2020 № 98 «Об утверждении Правил восстановительного лечения и медицинской реабилитации, в том числе детской медицинской реабилитации». Однако все перечисленные документы не содержат конкретных рекомендаций по срокам восстановления привычной активности по рассматриваемой нозологии.

Основным положительным результатом, полученным в ходе проведенного исследования, на наш взгляд, является не только обнаружение различий в подходах нейрохирургов, неврологов и реабилитологов к характеру и длительности восстановления пациентов после микродискэктомий, что укладывается в понятие «междисциплинарные разногласия», но и готовность разных специалистов к унификации рекомендаций на эту тему. Мы обратили внимание на то, что разброс ответов среди нейрохирургов оказался менее значимым, чем у других специалистов, что, возможно, обусловлено их работой в одном медицинском центре, полностью покрывающем нейрохирургическую потребность города. Наиболышие временные различия в рекомендациях (до 6 мес.) выявлены среди неврологов, что может быть следствием как наибольшей по ч́ислу респондентов выборки, так и работой в разных медицинских учреждениях. Все респонденты высказали заинтересованность в теме исследования, отмечали недостаточность исследований в данной области, а свои рекомендации основывали большей частью на собственном опыте и опыте коллег.

Анализ литературы, посвященной эффективности методов и сроков реабилитации после микродискэктомии на поясничном отделе позвоночника, в том числе систематических обзо- 


\begin{tabular}{|c|c|c|c|c|}
\hline Рекомендации & $\begin{array}{l}\text { Варианты } \\
\text { ответов }\end{array}$ & $\begin{array}{l}\text { Нейрохирурги } \\
\quad(\mathrm{n}=20)\end{array}$ & $\begin{array}{l}\text { Реабилитологи } \\
\quad(\mathrm{n}=12)\end{array}$ & $\begin{array}{c}\text { Неврологи } \\
(\mathrm{n}=28)\end{array}$ \\
\hline Продолжительность ограничения в положении сидя & $\begin{array}{l}2 \text { дня } \\
2 \text { нед. } \\
1 \text { мес. } \\
2 \text { мес. } \\
3 \text { мес. }\end{array}$ & $\begin{array}{c}12(60,0) \\
4(20,0) \\
4(20,0) \\
- \\
-\end{array}$ & \begin{tabular}{c}
$\quad-$ \\
\multicolumn{1}{c}{-} \\
$9(75,0)$ \\
$2(16,7)$ \\
$1(8,3)$
\end{tabular} & $\begin{array}{c}- \\
- \\
16(57,1) \\
8(28,6) \\
4(14,3)\end{array}$ \\
\hline Продолжительность ограничения лифтинговой нагрузки 5-10 кг & $\begin{array}{l}1 \text { мес. } \\
2 \text { мес. } \\
3 \text { мес. } \\
4 \text { мес. } \\
6 \text { мес. }\end{array}$ & $\begin{array}{c}8(40,0) \\
10(50,0) \\
- \\
2(10,0) \\
-\end{array}$ & $\begin{array}{c}- \\
1(8,3) \\
1(8,3) \\
4(33,4) \\
6(50,0)\end{array}$ & $\begin{array}{c}- \\
- \\
8(28,6) \\
9(32,1) \\
11(39,3)\end{array}$ \\
\hline Продолжительность ограничения вождения автомобиля & $\begin{array}{l}2 \text { нед. } \\
1 \text { мес. } \\
2 \text { мес. } \\
3 \text { мес. } \\
4 \text { мес. } \\
6 \text { мес. }\end{array}$ & $\begin{array}{c}4(20,0) \\
12(60,0) \\
4(20,0) \\
- \\
- \\
-\end{array}$ & $\begin{array}{c}- \\
4(33,3) \\
2(16,7) \\
- \\
6(50,0) \\
-\end{array}$ & $\begin{array}{c}- \\
2(7,1) \\
3(10,7) \\
19(67,9) \\
3(10,7) \\
1(3,6)\end{array}$ \\
\hline $\begin{array}{l}\text { Продолжительность ограничения глубоких наклонов и скручиваний } \\
\text { в позвоночнике }\end{array}$ & $\begin{array}{c}1 \text { мес. } \\
2 \text { мес. } \\
3 \text { мес. } \\
4 \text { мес. } \\
6 \text { мес. } \\
\text { Более } 6 \text { мес. }\end{array}$ & $\begin{array}{c}2(10,0) \\
16(80,0) \\
2(10,0) \\
- \\
- \\
-\end{array}$ & $\begin{array}{c}- \\
- \\
- \\
2(16,7) \\
8(66,6) \\
2(16,7)\end{array}$ & $\begin{array}{c}2(7,1) \\
6(21,5) \\
- \\
5(17,9) \\
13(46,4) \\
2(7,1)\end{array}$ \\
\hline Рекомендуемый срок возврата к сидячеи́ работе & $\begin{array}{l}\text { Через } 2 \text { мес. } \\
\text { Через } 4 \text { мес. } \\
\text { Через } 5 \text { мес. } \\
\text { Через } 6 \text { мес. }\end{array}$ & $\begin{array}{c}3(15,0) \\
16(80,0) \\
- \\
1(5,0)\end{array}$ & $\begin{array}{c}- \\
6(50,0) \\
5(41,7) \\
1(8,3)\end{array}$ & $\begin{array}{c}- \\
10(35,8) \\
9(32,1) \\
9(32,1)\end{array}$ \\
\hline Рекомендуемый срок возврата к физической работе & $\begin{array}{l}\text { Через } 2 \text { мес. } \\
\text { Через } 4 \text { мес. } \\
\text { Через } 5 \text { мес. } \\
\text { Через } 6 \text { мес. } \\
\text { Более } 6 \text { мес. }\end{array}$ & $\begin{array}{c}2(10,0) \\
14(70,0) \\
3(15,0) \\
1(5,0) \\
-\end{array}$ & $\begin{array}{c}\quad- \\
2(16,7) \\
4(33,3) \\
5(41,7) \\
1(8,3)\end{array}$ & $\begin{array}{c}- \\
2(7,1) \\
7(25,0) \\
18(64,3) \\
1(3,6)\end{array}$ \\
\hline Вид назначаемого корсета & $\begin{array}{l}\text { Полужесткий } \\
\text { Жесткий }\end{array}$ & $\begin{array}{r}16(80,0) \\
4(20,0)\end{array}$ & $\begin{array}{c}11(92,7) \\
1(8,3)\end{array}$ & $\begin{array}{r}19(67,9) \\
9(32,1)\end{array}$ \\
\hline $\begin{array}{l}\text { Продолжительность ношения ортопедического корсета } \\
\text { при физической нагрузке }\end{array}$ & $\begin{array}{l}1 \text { мес. } \\
2 \text { мес. } \\
3 \text { мес. } \\
4 \text { мес. } \\
6 \text { мес. }\end{array}$ & $\begin{array}{c}1(5,0) \\
2(10,0) \\
17(85,0) \\
- \\
-\end{array}$ & $\begin{array}{c}- \\
10(83,4) \\
1(8,3) \\
1(8,3) \\
-\end{array}$ & $\begin{array}{c}1(3,6) \\
3(10,7) \\
23(82,1) \\
- \\
1(3,6)\end{array}$ \\
\hline Продолжительность ограничения сексуальной активности & $\begin{array}{l}2 \text { нед. } \\
1 \text { мес. } \\
2 \text { мес } \\
3 \text { мес. } \\
6 \text { мес. }\end{array}$ & $\begin{array}{c}8(40,0) \\
10(50,0) \\
2(10,0) \\
- \\
-\end{array}$ & $\begin{array}{l}2(16,7) \\
2(16,7) \\
7(58,3) \\
1(8,3) \\
-\end{array}$ & $\begin{array}{c}1(3,6) \\
20(71,4) \\
4(14,3) \\
2(7,1) \\
1(3,6)\end{array}$ \\
\hline Даете ли вы письменные рекомендации по двигательному режиму? & $\begin{array}{l}\text { Всегда } \\
\text { Часто } \\
\text { Редко }\end{array}$ & $\begin{array}{r}12(60,0) \\
6(30,0) \\
2(10,0)\end{array}$ & $\begin{array}{c}5(41,7) \\
7(58,3) \\
-\end{array}$ & $\begin{array}{c}17(60,7) \\
11(39,3) \\
-\end{array}$ \\
\hline $\begin{array}{l}\text { Считаете ли вы полезным создание единых письменных } \\
\text { рекомендаций по двигательному режиму? }\end{array}$ & $\mathrm{Aa}$ & $20(100,0)$ & $12(100,0)$ & $28(100,0)$ \\
\hline $\begin{array}{l}\text { Согласны ли вы с предложенными рекомендациями и будете ли их } \\
\text { использовать в практической деятельности? }\end{array}$ & $\begin{array}{c}\text { Да } \\
\text { Частично } \\
\text { Нет }\end{array}$ & $\begin{array}{r}6(30,0) \\
12(60,0) \\
2(10,0)\end{array}$ & $\begin{array}{l}7(58,3) \\
4(33,4) \\
1(8,3)\end{array}$ & $\begin{array}{r}19(67,9) \\
6(21,4) \\
3(10,7)\end{array}$ \\
\hline
\end{tabular}


ров на эту тему 2006 г. и 2015 г., также выявил существенные различия как в подходах, так и в конкретных рекомендациях.

Установлено отсутствие существенных различий в оценке исходов реабилитационного лечения, касающихся снятия боли и последующей инвалидизации в условиях отсутствия двигательных ограничений или лимитирования нагрузки в сроки до одного года; при этом отсутствовал консенсус относительно необходимости, характера и сроков послеоперационных ограничений $[1,8]$.

Выявлены существенные различия между нейрохирургами Великобритании, Австралии, Италии и России, рекомендующими возвращение к повседневной двигательной активности пациентов в диапазоне от двух дней до 3 мес. [3-6].

Опрошенные в 2012 г. специалисты центров Великобритании предоставляли пациентам письменные рекомендации, которые, однако, отличались друг от друга из-за того, что разрабатывались самостоятельно в каждом учреждении [9]. Послеоперационные ограничения устанавливались врачами исходя из теоретических соображений, собственной практики и существующих в пределах медицинского центра разработок, а не на основе имеющихся публикаций.

Выявлены слабые доказательства пагубных последствий исключения каких-либо послеоперационных ограничений, а также того, что большинство рекомендаций по лимитированию сроков возвращения к повседневной активности замедляют восстановление и возвращение к работе: поощрение мобильности и, по возможности, скорейшее возвращение к полной деятельности после операции облегчают боль и способствуют раннему возвращению к работе $[10,11]$.

Отмечено также, что рекомендации по ограничению нагрузки и возвращению пациентов к предыдущим обязанностям с учетом их индивидуальных особенностей могут быть полезны, в том числе с юридической точки зрения [12]. Одновременно отмечено, что диссонанс в рекомендациях тяжело сказывается на пациентах, которые не уверены в том, какие действия они могут или должны предпринять после операции [13].

\section{Заключение}

Проведенное исследование продемонстрировало существенные междисциплинарные различия в отноше- нии тактики ведения пациентов после поясничной микродискэктомии врачами разных специальностей. Характерно, что такой диссонанс соответствует мировым тенденциям, причем в анкетированной нами группе выявлены несколько бо́льшие сроки, рекомендуемые для возвращения пациентов к повседневной двигательной активности относительно рекомендаций, используемых врачами других стран.

Исследование показало целесообразность разработки междисциплинарных рекомендаций по реабилитационно-восстановительному лечению пациентов после микродискэктомии, основой для которых могут быть рекомендации, предложенные авторами ранее и в основном поддержанные респондентами [7].

С целью разработки единой тактики послеоперационного лечения и реабилитации пациентов после поясничной микродискэктомии необходимы дальнейшие исследования с высокой степенью доказательности по рекомендуемым срокам ограничения двигательной активности.

Исследование не имело спонсорской поддержки. Авторы заявляют об отсутствии конфликта интересов.

\section{Литература/References}

1. Oosterhuis T, Costa LO, Maher CG, de Vet HC, van Tulder MW, Ostelo RW. Rehabilitation after lumbar disc surgery. Cochrane Database Syst Rev. 2014;(3):CD003007. DOI: 10.1002/14651858.CD003007.

2. Gibson JN, Waddell G. Surgery for degenerative lumbar spondylosis. Cochrane Database Syst Rev. 2005;(2):CD001352. DOI: 10.1002/14651858.CD001352.

3. McGregor AH, Dicken B, Jamrozik K. National audit of post-operative management in spinal surgery. BMC Musculoskelet Disord. 2006;7:47. DOI: 10.1186/1471-2474-7-47.

4. Daly CD, Lim KZ, Ghosh P, Goldschlager T. Perioperative care for lumbar microdiscectomy: a survey of Australasian neurosurgeons. J Spine Surg. 2018;4:1-8. DOI: $10.21037 /$ iss.2018.01.03.

5. Zoia C, Bongetta D, Poli JC, Verlotta M, Pugliese R, Gaetani P. Intraregional differences of perioperative management strategy for lumbar disc herniation: is the Devil really in the details? Int J Spine Surg. 2017;11:1. DOI: 10.14444/4001.

6. Кащеев А.А., Гуща А.О., Арестов С.о. Общие принципы лечения и реабилитации пациентов после операций по поводу дегенеративно-дистрофических поражений пояснично-крестцового отдела позвоночника // РМж. 2012. № 31. C. 1548. [Kashcheev AA, Gushcha AO, Arestov SO. General principles for the treatment and rehabilitation of patients after surgery for degenerative-dystrophic lesions of the lumbosacral spine. RMJ. 2012;31:1548-1551. In Russian].

7. Муратбекова ШІ.С., Шевелева Н.И., Турсынов Н.И. Рекомендации для пациентов в раннем восстановительном периоде после микродискэктомии на поясничном отделе позвоночника // Свидетельство о внесении сведений в государственный реестр прав на объекты, охраняемые авторским правом Республики Казахстан № 3615 от 27 мая 2019 г. [Muratbekova ShS, Sheveleva NI, Tursynov NI. Recommendations for patients in the early recovery period after microdisectomy in the lumbar spine. Certificate on entering information into the State Register of Rights to Objects Protected by Copyright of the Republic of Kazakhstan No. 3615 dated May 27, 2019. In Russian].

8. Rushton A, Wright C, Goodwin P, Calvert M, Freemantle N. Physiotherapy rehabilitation post first lumbar discectomy: a systematic review and meta-analysis of randomised controlled trials. Spine. 2011;36:E961-E972. DOI: 10.1097/BRS.0b013e3181f0e8f8.

9. Goodwin PC, Wright CC, Allan C, Crowther L, Darley C, Heap A, Paul E, White L, Rushton A. Evidence-based development of a post-surgical lumbar discectomy leaflet intervention: a Delphi consensus study. BMJ Open. 2015;5:e006069. DOI: 10.1136/ bmjopen-2014-006069. 
10. Kjellby-Wendt G, Styf J, Carlsson SG. Early active rehabilitation after surgery for lumbar disc herniation: a prospective, randomised study of psychometric assessment in 50 patients. Acta Orthop Scand. 2001;72:518-524. DOI: 10.1080/000164701753532871.

11. Kjellby-Wendt G, Carlsson SG, Styf J. Results of early active rehabilitation 5-7 years after surgical treatment for lumbar disc herniation. J Spinal Disord Tech. 2002;15:404409. DOI: 10,1097/00024720-200210000-00010.

\section{Адрес для переписки:}

Турсынов Нуртас Исатаевич

M01K6T3, Казахстан, Караганда, ул. Гоголя, 40,

Медицинский университет Караганды,

nurtasisataevich@mail.ru

Статья поступила в редакиию 13.02.2020

Рецензирование пройдено 08.05.2020

Подписано в печать 12.05.2020
12. Pope MH, Magnusson ML, Wilder DG, Goel VK, Spratt K. Is there a rational basis for post-surgical lifting restrictions? 2. Possible scientific approach. Eur Spine J. 1999;8:179-186. DOI: $10.1007 / 5005860050154$.

13. Christensen FB, Laurberg I, Bunger CE. Importance of the back-caf concept to rehabilitation after lumbar spinal fusion: a randomised clinical study with a 2 year follow up. Spine. 2003;28:2561-2569. DOI: 10.1097/01.BRS.0000097890.96524.A1.

Address correspondence to:

Tursynov Nurtas Isataevich

Karaganda Medical University,

40 Gogol str., Karaganda, M01K6T3, Kazakhstan,

nurtasisataevich@mail.ru

Received 13.02.2020

Review completed 08.05.2020

Passed for printing 12.05.2020

Нуртас Исатаевич Турсынов, д-р мед. наук, ассоциированный профессор кафедры неврологии, нейрохирургии, психиатрии и реабилитологии, Медицинский университет Караганды, Казахстан, М01КбТЗ, Караганда, ул. Гоголя, 40, ORCID: 0000-0003-1939-7506, Tursynov@qmu.kz;

Марина Арчиловна Григолашвили, канд. мед. наук, зав. кафедрой неврологии, нейрохирургии, психиатрии и реабилитологии, Медищинский университет Караганды, Казахстан, М01КбТЗ, Караганда, ул. Гоголя, 40, ORCID: 0000-0001-6722-1762, Grigolashvili@qmu.kz;

Наиля Игоревна Шевелева, д-р мед. наук, профессор кафедры неврологии, нейрохирургии, психиатрии и реабилитологии, Медищинскии университет Караганды, Казахстан, М01К6Т3, Караганда, ул. Гоголя, 40, ORCID: 0000-0002-6658-7399, n_sheveleva@qтu.kz;

Шынар Сабитовна Муратбекова, ассистент кафедры неврологии, нейрохирургии, психиатрии и реабилитологии, Медицинкий университет Караганды, Казахстан, М01К6ТЗ, Караганда, ул. Гоголя, 40, ORCID: 0000-0002-4657-4021, MuratbekovaS@qти.kz.

Nurtas Isataevich Tursynov, DMSci, associate professor of the Department of Neurology, Neurosurgery, Psychiatry and Rehabilitation, Karaganda Medical University, 40 Gogol str., Karaganda, M01K6T3, Kazakbstan, ORCID: 0000-0003-1939-7506,Tursynov@qmu.kz;

Marina Archilouna Grigolashvili, MD, PbD, Head of the Department of Neurology, Neurosurgery, Psychiatry and Rehabilitation, Karaganda Medical University, 40 Gogol str., Karaganda, M01K6T3, Kazakbstan, ORCID: 0000-0001-6722-1762, Grigolashvili@qmu.kz;

Naila Igorevna Sheveleva, DMSci, Professor of the Department of Neurology, Neurosurgery, Psychiatry and Rehabilitation, Karaganda Medical University, 40 Gogol str., Karaganda, M01K6T3, Kazakbstan, ORCID: 0000-0002-6658-7399, n_sheveleva@qmu.kz;

Shynar Sabitouna Muratbekova, teaching assistant of the Department of Neurology, Neurosurgery, Psychiatry and Rebabilitation, Karaganda Medical University, 40 Gogol str., Karaganda, M01K6T3, Kazakbstan, ORCID:0000-0002-4657-4021, MuratbekovaS@qmu.kz. 